International Journal of Translational

Medical Research and Public Health (2020), Volume 4, Issue I, 50-55

\begin{tabular}{|c|c|c|}
\hline & & $\begin{array}{l}\text { INTERNATIONAL JOURNAL OF TRANSLATIONAL } \\
\text { MEDICAL RESEARCH AND PUBLIC HEALTH } \\
\text { ISSN 2576-9502 (Online) }\end{array}$ \\
\hline I]TMRPH & Available online at www.ijtmrph.org & $\begin{array}{l}\text { ISSN 2576-9499 (Print) } \\
\text { DOI: 10.21 106/ijtmrph. I } 30\end{array}$ \\
\hline
\end{tabular}

\title{
SHORT RESEARCH COMMUNICATION | DIABETES MELLITUS Gestational Diabetes Mellitus and Associated Health Risk Outcomes in Mothers and Neonates
}

\author{
Maureen Atieno Adoyo, $\mathrm{PhD} ;{ }^{\square}$ Yeri M. Kombe, $\mathrm{PhD}^{2}$ \\ 'Institute of Tropical Medicine - KEMRI, PO Box 54840, Nairobi, KENYA; ${ }^{2}$ Kenya Medical Research Institute, PO Box 54840, Nairobi, KENYA, \\ ¿Corresponding author email: lornaloo.adoyo@gmail.com
}

ABSTRACT

Global Gestational Diabetes Mellitus (GDM) pregnancy complication is estimated at 200,000 cases annually with serious health consequences for maternal and neonates. A cohort study to establish GDM risks and associated health outcomes was conducted among 238 women attending antenatal clinic in selected health facilities in Nairobi, Kenya. Results indicated that mothers with GDM are four times more likely to have varied physical discomforts and complicated deliveries as result of high neonatal birth weight. To improve population health, there is need to advocate for inclusion of diabetes testing and management as part of Essential Health Services Package for Kenya to realize positive health outcomes among women.

Key words: • Gestational diabetes mellitus • Maternal • Neonates

Copyright () 2020 Adoyo et al. Published by Global Health and Education Projects, Inc. This is an open-access article distributed under the terms of the Creative Commons Attribution License CC BY 4.0.

\section{Introduction}

Gestational Diabetes Mellitus (GDM), defined as any degree of glucose intolerance with onset or first recognition during pregnancy, is one of the health conditions that very little attention has been paid to until a decade ago, when there was notable increase of non- communicable diseases cases globably. The prevalence of GDM is estimated to be in the range of I to $14 \%$ of all pregnancies, depending on the population characteristics being studied and the diagnostic technique' Global GDM Pregnancy complications are estimated at 200,000 annually. ${ }^{2}$ Further, the outcome of GDM in population is of greater magnitude touching on maternal and neonatal health. The associated conditions are: physical discomfort associated with type 2 diabetes in maternal while for their offsprings the risks may include fetal macrosomia, hypoglycemia, jaundice, polycythaemia, obesity and diabetes in late adolescence or young adulthood.

Perinatal mortality risks associated with GDM is mostly attributable to undiagnosed type 2 diabetes which is common among mothers with obesity. Other complications such as congenital malformation in neonates is slightly increased in infants of mothers with GDM whether untreated, moderate or severe compared to the general population, it is worth noting that GDM prevalence has increased by 
approximately $10-100 \%$ in the last 20 years. $^{3}$ This is a reflection of the patterns of increasing diabetes and obesity conditions in the population. Systematic review done in Africa also indicated that little is known about GDM in Africa and highlights the need for further research. ${ }^{3}$

Kenya, continues to report worrying statistics in Non-Communicable Diseases (NCDs), diabetes included, and the latest diagnosis in much younger population underscores the need for an investigation to be conducted to identify predisposing factors that need modifications in order to realize improvements in health outcomes for mothers and neonates. The sudden upsurge of diabetes among Kenya's young population is estimated at about I.8 million people living with diabetic condition. ${ }^{4}$ The souring numbers and the latest diagnosed case in infant and being the youngest case that has ever been recorded in Kenya, has pushed the bar for an investigation to be conducted and make information available for modification of predisposing factors especially during pregnancy. Reviewed studies also indicate that there is likelihood that future generations have chances of developing obesity and other non-communicable health problems that are mostly determined in the womb hence the interest in studying gestational diabetes and the associated health risks. ${ }^{5}$ Evidence-based information on gestational diabetes and associated perinatal health risks in Kenyan context is likely to increase acceptability of research findings that will go a long way in informing the general public on issues related to nutrition and lifestyle at pregnancy and after child birth giving rise to desirable health outcomes among maternal and neonates. The objectives of this study were to examine risks and health outcomes among mothers with GDM mothers compared to non-GDM mothers.

\section{Methods}

This Cohort study was conducted to establish risks and possible outcomes in GDM condition among pregnant women. Nairobi County was considered as study site on the basis of being populous city with population estimate of 3 million people ${ }^{6}$ and having desirable characteristics in relation to urbanization and sedentary lifestyle, pointed as key drivers to the evolution of NCDs epidemic in developing countries.

\section{I. Study Population, Sample Size and Inclusion Criteria}

The study population comprised of women in their third trimester of pregnancy and sample size was determined for the two groups (GDM and nonGDM) with $10 \%$ effect size at $95 \%$ confidence Interval $(\mathrm{Cl})$, the existing high birth weight among GDM group was estimated at $13 \%$ and $23 \%$ among non GDM group. To factor in potential attrition, the obtained sample of 23I participants was then adjusted by $10 \%$ to 254 for mother-baby pair. Simple random technique was used to select 10 facilities from the Master Facility List (MFL).

\subsection{Study Procedure and Data Collection}

Oral Glucose Test (OGT) was conducted to ascertain GDM condition and also for categorization into GDM and non-GDM groups necessary for making comparison. Participants were required to continue with normal diet one hour leading up to test while avoiding bathroom visits before the procedure.After blood sample collection, participants were instructed to have sweet drink or concentrated glucose solution within five minutes after which glucose levels were tested again. Structured questionnaires were also administered in three phases to assess initial eligibility, record prevailing physical condition during pregnancy and follow up interview within 28 days after giving birth. Figure I shows the methodological diagram of the study.

\subsection{Statistical Analysis}

Using Stata version $13^{7}$ risk factors were analysed as Odds Ratio and univariate logistic regression analysis was conducted for nominal, ordinal and interval variables such as $\mathrm{BMI}$, age, and birth weight, respectively. Multilevel logistic regression was applied to account for confounding variable by analysing GDM and Non- GDM independent variables in order to predict health outcomes alternately in mothers and neonates.

\subsection{Ethical Approval}

Ethical Review Committee of Kenyatta National Hospital in Conjunction with the University of 


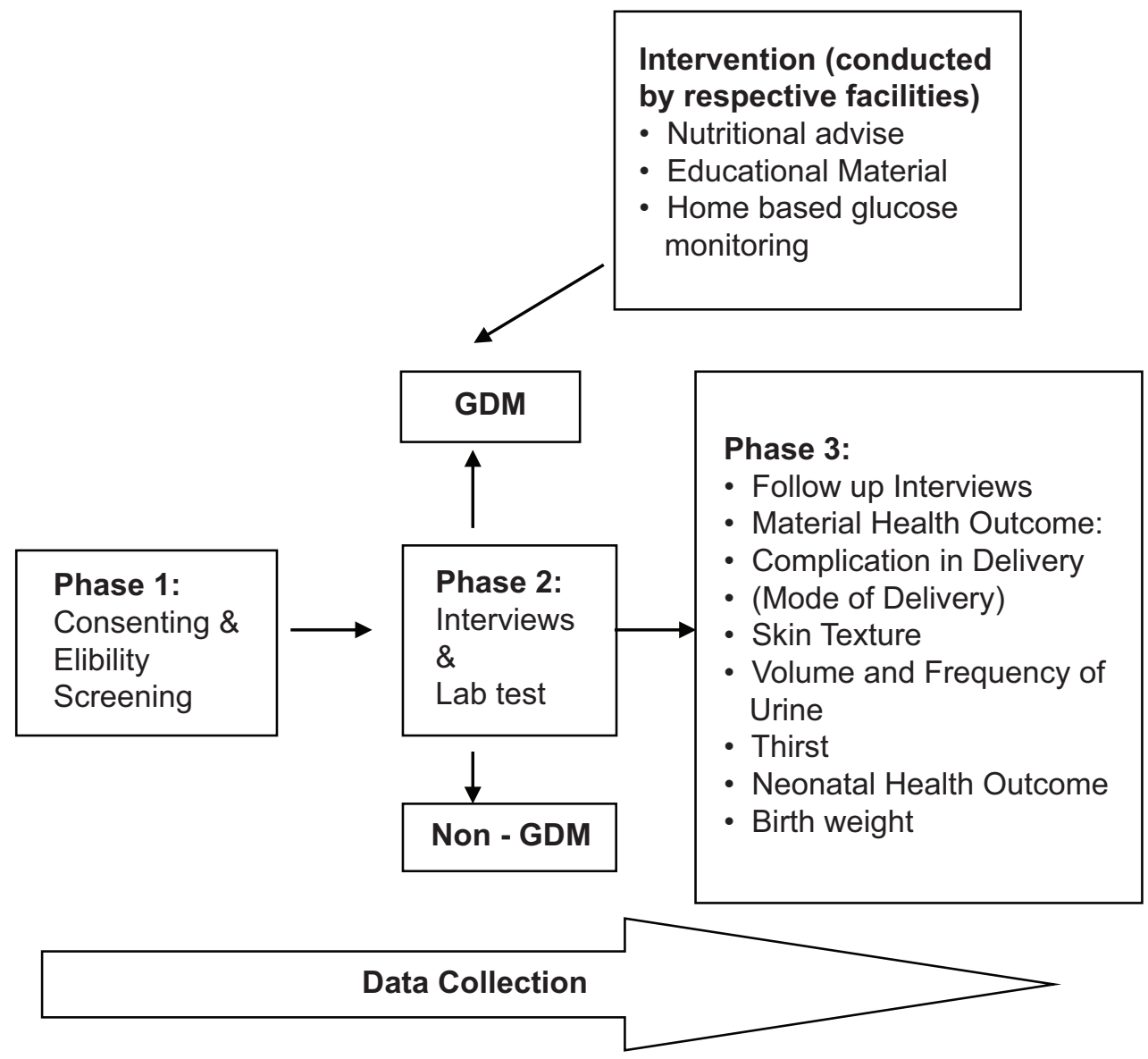

Figure I: Methodological diagram

Nairobi verified the conformation of research to ethical requirements in handling human subjects. Written permission was obtained from local authorities and management of the selected maternity facilities. Additionally, study participants were required to consent to participate in the study. Confidentiality was maintained by assigning participants unique codes and resulting information was solely used for study purposes and recommendation towards Maternal and Child Health Intervention Programs, including policy changes that were intended for the benefit of population in Kenya and the region. Investigators engaged in the study underwent certification process by ethically certified scientific body - CITI and all ethical practices were adhered to. ${ }^{8}$

\section{Results}

Overall, the study had 238 participants who completed the follow-up process; out of these responses 66 [27.73\%] had GDM. The analysis of trend showed that mothers with family history of diabetes had twice the risk of developing GDM $[O R=2.27 ; 95 \%$ C.I:I.23-4.17] in comparison with mothers who did not have family history of diabetes. Additionally, body mass index (BMI) status showed positive association with GDM, with findings indicating that obese mothers had double-fold increased risk of developing GDM [OR $=28 ; 95 \% \mathrm{Cl}$ : I I.83-36.7]. Similarly, age showed positive association with GDM condition in mothers [OR = I.15; $95 \%$ Cl:I.089-I.206]. 
Table I: Demographic characteristics of participants and associated health outcomes in GDM and NonGDM group

\begin{tabular}{|c|c|c|c|c|}
\hline Indicator & GDM $(n=66) \%$ & Non-GDM $(n=172) \%$ & OR (95 Cl) & P-value \\
\hline \multicolumn{5}{|l|}{ Diabetic History } \\
\hline No diabetic history (Ref.) & 62.3 & 42.11 & 1.00 & \\
\hline Had diabetic history & 37.7 & 57.89 & $2.27(1.23,4.17)$ & 0.008 \\
\hline \multicolumn{5}{|l|}{ BMI before pregnancy } \\
\hline Underweight (Ref.) & 1.5 & 7.74 & 1.00 & \\
\hline Normal & 19.70 & 56.55 & $1.78(0.214,14.74)$ & 0.593 \\
\hline Overweight & 54.55 & 35.12 & $7.93(0.99,63.22)$ & 0.051 \\
\hline Obese & 24.24 & 0.06 & $2.08(1.83,3.65)$ & 0.000 \\
\hline Extreme obese & 0.0 & 0 & & \\
\hline Mean age & $(31.59,34.32)$ & $(27.01,28.78)$ & $1.15(1.089,1.206)$ & 0.000 \\
\hline \multicolumn{5}{|l|}{ Urine frequency per day } \\
\hline$<=2$ hours (Ref.) & $|2.3|$ & 18.35 & 1.00 & \\
\hline 3-4 hours & 53.85 & 70.25 & $0.99(053,1.87)$ & 0.983 \\
\hline$>=4$ hours & 33.85 & 11.39 & $1.90(0.81,4.46)$ & 0.141 \\
\hline \multicolumn{5}{|l|}{ Volume of urine per day } \\
\hline Little (Ref.) & 21.54 & 39.75 & 1.00 & \\
\hline Normal* & 52.31 & 46.58 & $2.07(1.02,4.19)$ & 0.043 \\
\hline A lot* & 26.15 & 13.66 & $3.53(1.50,8.33)$ & 0.004 \\
\hline \multicolumn{5}{|l|}{ Skin texture } \\
\hline Soft and glowing (Ref.) & 24.62 & 47.17 & 1.00 & \\
\hline Dry and rough & 75.38 & 52.83 & $0.37(0.19,0.69)$ & 0.002 \\
\hline \multicolumn{5}{|l|}{ Blood pressure } \\
\hline Normal (Ref.) & 61.67 & 18.35 & 1.00 & \\
\hline High & 38.33 & 70.25 & $2.53(1.29,4.94)$ & 0.007 \\
\hline Low & 0.0 & 11.29 & & \\
\hline \multicolumn{5}{|l|}{ Neonates birth weight } \\
\hline Underweight $<2.5 \mathrm{Kg}$ (Ref.) & 1.52 & 7.65 & 1.00 & \\
\hline Small $2.6 \mathrm{Kg}-3.5 \mathrm{Kg}$ & 31.82 & 57.65 & $2.78(0.35,22.47)$ & 0.336 \\
\hline High birth weight* & 66.67 & 34.71 & $9.69(0.01,0.59)$ & 0.013 \\
\hline
\end{tabular}

In relation to physical discomfort, results indicated that mothers with GDM were four times likely to experience high frequency of urination $[O R=4.33$; 95\% C.I: I.I4-16.47], produced three times higher volume of urine [OR = 3.65; 95\% C.I: I.I2-II.93], while episiotomy mode of delivery [OR $=0.29 ; 95 \%$ $\mathrm{Cl}: 0.10-0.85]$ and skin texture $[\mathrm{OR}=0.17 ; 95 \%$ $\mathrm{Cl}: 0.077$ - 0.385] showed negative association but remained significantly common among GDM mothers [P-value <0.05]. Similarly, high blood pressure was more common 23 [38.89\%] among
GDM mothers compared to 27 [18.75\%] among non-GDM mothers.

Neonatal birth weight was nine times higher among GDM mothers [OR $=9.69 ; 95 \%$ C.I: 0.01 - 0.59]. Consequently, the association of GDM and high neonatal birth weight was also highly significant $[\mathrm{P}$ - value=0.0I3]. Caesarean Section (CS) mode of delivery for present pregnancy was high 38 [58.46\%] among mothers with GDM compared to 60 [35.55\%] for mothers with non-GDM condition. 
Table I shows demographic characteristics of participants and associated health outcomes in GDM and Non-GDM group.

\section{Discussion}

The probability of GDM mothers developing type 2 diabetes after birth was highly significant hence the findings rejected the null hypothesis that there is no difference in maternal health outcome between women with GDM and those without GDM. Symptoms related to diabetic condition such feeling thirsty, high frequency and large volume of urine in GDM mothers was significantly expressed during pregnancy and persistent after delivery. This study results are consistent with a study done by Ferrara and the American Diabetes Association indicating that frequency of GDM usually reflects type 2 diabetes in the underlying population with more that $50 \%$ developing type 2 diabetes within $5-10$ years after delivery. ${ }^{9}$

High neonatal birth weight was found to be positively associated with GDM condition in mothers, with estimated cases to be 44 [66.67\%] compared to neonates delivered by non-GDM mothers 59 [34.7I\%]. High birth weight phenomena also referred as macrosomia was observed in another report which showed a positive correlation between maternal blood glucose levels and increased birth weight.' Findings in other studies also give indication that women with GDM are more likely to give birth to macrosomia or large-for-gestational-age infants resulting to complications such as obstructed labor, a birth injury for the infants and the death of the mother or the baby or both.

\section{I. Limitations}

A much larger sample size given the high birth rate in the country would have been much desirable for any robust cohort study. However, due to limitation in time and material resources the study was restricted to 6 sub-Counties of Nairobi with a total of 10 facilities out of the listed 97 being selected for the study. This also meant that it took a long time to achieve minimum desirable and statistically adequate sample.
Loss-to follow up was evident when mothers failed to enrol for Post-Natal Care (PNC) either because they sought for well-baby clinic elsewhere outside selected study facilities or other unknown reason as they could not be traced via telephone and neither did the study had allocation to trace mothers to their residences. To make the study finding generalizable at acceptable levels, attrition was catered for by adjust sample size by $10 \%$ upwards.

Study had standardization limitations in OGT as the study relied on different laboratories where participants chose to conduct their tests. There is possibility that the different settings might have affected precision of the tests thus, the researchers recommend the inclusion of screening resources in future to improve accuracy in test results and effectiveness of research process. In order to establish true prevalence and the impact of GDM, there is need to conduct a study with robust methodological approach using standardized and optimal diagnosis procedures for GDM and recruitment of adequate sample size with a longer follow up period of between 2-5 years after delivery.

\section{Conclusion and Implications for Translation}

Elevated blood glucose in pregnant mothers, also known as GDM, is a precursor to complications during pregnancy and may result in obstructed labor leading to caesarean section birth due to increased chances of having large gestational weight in neonates.

\section{Compliance with Ethical Standards}

Conflicts of Interest: The authors declare that they have no conflict of interest. Financial Disclosure: This study was solely funded by individual resources. Funding/ Support: None. Ethics Approval: Ethical review was given by Scientific Commitee of Kenyatta National Hospital/Unversity of Nairobi and relevant approvals were issued. Acknowledgements: The authors thank the management of health facilities and participants. Dr. Adoyo acknowledges the professionalism of Ethics Committee and thanks her mentors, Dr.Yeri Kombe and Professor Charles Mbakaya. Special tribute to Prof.Venny Nyambati who succumbed to cancer just before the completion of the study project. 


\section{Key Messages}

- Testing and managing Gestational Diabetes is important for ensuring a healthy mother and baby because a healthy pregnant mother is important for a healthy population.

- Proper nutrition and healthy lifestyle during pregnancy is necessary for obtaining desirable health outcomes in mothers and their neonates.

\section{References}

I. Society for Maternal-Fetal Medicine. High-Risk Pregnancy Care, Research, and Education for Over 35 Years.; 2010. http://www.mfmnyc.com/wp-content/ uploads/20I5/I0/SMFMMonograph3.I.pdf. Accessed May 4, 2020.

2. Yvette C. Terrie. Gestational Diabetes: What Patients Need to Know. Pharmacy Times. http:// www.pharmacytimes.com/publications/issue/2009/ october2009/counselinggestationaldiabetes-1009. Published 2015.Accessed February 15, 2017.

3. Macaulay S, Dunger DB, Norris SA. Gestational diabetes mellitus in Africa: a systematic review. PLoS One. 20I4;9(6):e9787I. Published 2014 Jun 3.
doi:I0.137|/journal.pone.009787I.

4. Ayah R, Joshi MD, Wanjiru R, et al.A population-based survey of prevalence of diabetes and correlates in an urban slum community in Nairobi, Kenya. BMC Public Health. 20 I3; I3(I):37I. doi: I0.I I86/I47I-2458-13-37I

5. Chu SY, Callaghan WM, Kim SY, et al. Maternal obesity and risk of gestational diabetes mellitus. Diabetes Care. 2007;30(8):2070-2076. doi:10.2337/dc06-2559a.

6. Kenya National Bureau of Statistics (KNBS). The 2009 Kenya Population and Housing Census - Population Distribution by Age, Sex and Administrative Units. 2010;IC:546. http://www.knbs.or.ke/index. php?option=com_phocadownload\&view=category\&d ownload=584: volume- I c-population-distribution-byage-sex-and-administrative-units\&id=109:populationand-housing-census-2009\&ltemid $=599$.

7. StataCorp. 2013. Stata Statistical Software: Release I3. College Station, TX: StataCorp LP.

8. CITI Program: Collaborative Institutional Training Initiative. https://www.citiprogram.org. Accessed May 4, 2020.

9. SattarN,GreerIA.Pregnancycomplicationsandmaternal cardiovascular risk: opportunities for intervention and screening? BMJ. 2002;325(7356):I57-I60.

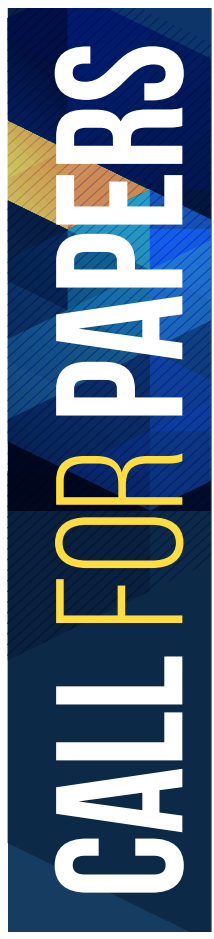

\section{TWO JOURNALS, ONE PRIORITY:}

The Global Health and Education Projects, Inc., Washington, DC. USA, is calling for high-quality submissions for publication in its two flagship, high-impact, international journals. Submissions from interdisciplinary researchers with the potential to advance science, increase understanding, improve population health, and save lives, are welcome.

\section{INTERNATIONAL JOURNAL OF MCH AND AIDS | IJMA}

IJMA is a multidisciplinary, peer-reviewed, open-access, global health journal that publishes original research articles, review articles, field studies, and commentaries in all areas of pediatrics, maternal and child health, (MCH), human immunodeficiency virus (HIV) and acquired immune deficiency disease (AIDS). IJMA covers all aspects of women's health, child health, men's health, family health, HIV/AIDS, and more.

JOURNAL WEBSITE / FREE ONLINE SUBMISSION: www.mchandaids.org.

EMAIL FOR QUESTIONS OR INQUIRIES: submissions@mchandaids.org.

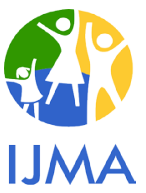

\section{INTERNATIONAL JOURNAL OF TRANSLATIONAL MEDICAL RESEARCH AND PUBLIC HEALTH I IJTMRPH}

IJTMRPH is an interdisciplinary, peer-reviewed, open-access journal that publishes original research articles, review articles, field studies, and commentaries on all aspects of applied or translational public health, global health, and medical research. IJTMRPH covers all aspects of public health and medical research, including Epidemiology, Environmental health, Occupational health, Community health, and more.

JOURNAL WEBSITE / FREE ONLINE SUBMISSION: www.ijtmrph.org. EMAIL FOR QUESTIONS OR INQUIRIES: submissions@ijtmrph.org.

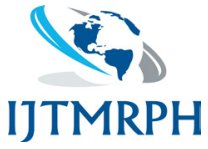

